\title{
Aflatoxins and $A$. flavus Reduction in Loaf Bread through the Use of Natural Ingredients
}

\author{
Juan M. Quiles ${ }^{1,+}$, Raquel Torrijos ${ }^{1,+}$, Fernando B. Luciano ${ }^{2}$, Jordi Mañes ${ }^{1}$ and \\ Giuseppe Meca ${ }^{1, *}$ \\ 1 Laboratory of Food Chemistry and Toxicology, Faculty of Pharmacy, University of Valencia, \\ Av. Vicent Andrés Estellés s/n, 46100 Burjassot, Spain; juan.quiles@uv.es (J.M.Q.); tocara@alumni.uv.es (R.T.); \\ jorge.manes@uv.es (J.M.) \\ 2 School of AgriculturalSciences and Veterinary Medicine, PontifíciaUniversidade Católica do Paraná, \\ BR 376 Km 14, 83010-500 São José dos Pinhais, Brazil; fernando.luciano@pucpr.br \\ * Correspondence: giuseppe.meca@uv.es; Tel.: +34-963544959; Fax: +34-96354954 \\ + These authors contributed equally to this work.
}

Received: 6 June 2018; Accepted: 3 July 2018; Published: 4 July 2018

\begin{abstract}
In this study, the antifungal activity of yellow mustard (YMF) and oriental mustard (OMF) meal extracts against 14 strains of fungi was tested on a solid medium. The results obtained with the YMF were next confirmed in liquid medium determining the minimum inhibitory concentration (MIC) and the minimum fungicide concentration (MFC). Finally, the use of YMF as a natural preservative to extend the useful life of bread was evaluated. Breads with different concentrations of YMF (2, 4, 6 and $8 \mathrm{~g} / \mathrm{kg})$ were prepared and contaminated with Aspergillus flavus ISPA 8111 and Penicillium nordicum CECT 2320. For 10 days the formation of mycelium was observed, and after that the fungal growth and the mycotoxins production was determined. The results obtained with the YMF were compared with breads treated with the commercial additive sodium propionate (E-281). The results showed a significant reduction of the fungal population using $6 \mathrm{~g} / \mathrm{kg}$ and $8 \mathrm{~g} / \mathrm{kg}$ of YMF in bread contaminated with $A$. flavus and with $P$. nordicum and an extensions of the breads shelf life of 7 and 5 days, respectively, in comparison with the control experiment. A reduction of $78 \%$ of $\mathrm{AFB}_{1}$ was observed using $6 \mathrm{~g} / \mathrm{kg}$ of YMF while no $\mathrm{AFB}_{1}$ production was detected employing $8 \mathrm{~g} / \mathrm{kg}$ of YMF in bread preparation.
\end{abstract}

Keywords: aflatoxins; shelf life; mustard flour; mycotoxin reduction; LC-MS/MS

\section{Introduction}

Aflatoxins (AFs) (Figure 1) are difuranocoumarin derivatives mainly produced through the polyketide pathway by two species of Aspergillus fungi which are especially found in areas with hot and humid climates. Aspergillus flavus is ubiquitous in Nature, preferring the colonization of the aerial parts of the plants (leaves, flowers) and usually producing group B AFs. Aspergillus parasiticus which produces both $B$ and $\mathrm{G}$ AFs, is more adapted to soil environments and has more limited distribution [1]. Aspergillus bombycis, Aspergillus ochraceoroseus, Aspergillus nomius, and Aspergillus pseudotamari are also AF-producing species, but they are found less frequently [2].

The four main $\mathrm{AFs}_{\text {are }} \mathrm{AFB}_{1}, \mathrm{AFB}_{2}, \mathrm{AFG}_{1}$ and $\mathrm{AFG}_{2}$. They can directly contaminate agricultural products and other foodstuffs under pre-and post-harvest conditions. $\mathrm{AFB}_{1}$ is usually predominant in crops as well as in food products and it has been found to exhibit the greatest toxigenic potential [3]. The AFs can be classified from highest to lowest toxicity in the following order: $\mathrm{AFB}_{1}, \mathrm{AFG}_{1}, \mathrm{AFB}_{2}$ and $\mathrm{AFG}_{2}$, and this is probably explained by the presence of epoxidation at the 8,9 double bond, as well as by the greater power that accompanies the ring. $\mathrm{AFB}_{1}$ is one of the most potent toxic carcinogens, 
it is a teratogen and a mutagen and it is listed as a Group I carcinogen by the International Agency for Research on Cancer (IARC) because it is a cause of human primary hepatocellular carcinoma [4].<smiles>COc1cc2c(c3oc(=O)c4c(c13)CCC4=O)[C@H]1C=CO[C@H]1O2</smiles>

AFB1<smiles>COc1cc2c(c3oc(=O)c4c(c13)CCC4=O)[C@H]1CCO[C@@H]1O2</smiles>

AFB2<smiles>COc1cc2c(c3oc(=O)c4c(c13)CCOC4=O)[C@H]1C=CO[C@@H]1O2</smiles><smiles>COc1cc2c(c3oc(=O)c4c(c13)CCOC4=O)[C@H]1CCO[C@H]1O2</smiles>

Figure 1. Chemical structures of the aflatoxins.

Plants belonging to the Brassicaceae family are known worldwide for their rich bioactive composition, highlighted by glucosinolates, which are cleaved by an enzymatic reaction to give isothiocyanates (ITCs). These ITCs possess many properties, among which their biocidal activity (fungicidal, bactericidal and on insects and small invertebrates), as well as herbicidal, antioxidant and anticancer effects may be highlighted [5]. Both Sinapis alba (yellow or white mustard) and Brassica juncea (brown and oriental mustard) contain high levels of glucosinolates, which are cleaved by myrosinase (EC 3.2.1.147) in the presence of moisture, forming ITCs plus thiocyanates, nitriles and some other minor compounds. In oriental mustard, myrosinase forms allyl isothiocyanate (AITC) from the main glucosinolate, sinigrin. Its effectiveness inhibiting the development of fungi [6,7], as well as the bacteria Escherichia coli O157: H7 [8], Listeria monocytogenes [9] or Salmonella sp. [10] has been proven.

In yellow mustard, the ITC that is formed is $p$-hydroxybenzylisothiocyanate $(p$-HBIT) from the main glucosinolate sinalbine. Ekanayake et al. [11] demonstrated that $p$-HBIT had significant antimicrobial activity against several foodborne pathogens, including Escherichia coli, Staphylococcus aureus, Campylobacter jejuni, Pseudomonas aeruginosa, Salmonella enteritidis, Listeria monocytogenes, Shigella boydii and Clostridium spp.

The aim of the study was to evaluate the use of oriental and yellow mustard flours to reduce contamination by toxigenic fungi and their mycotoxins. First, the antifungal activity of yellow mustard flour (YMF) and oriental mustard meal (OMF) was studied against fourteen different fungal species. Next, the reduction of fungal growth, the formation of mycotoxins and the YMF improvement of the useful life of moldy bread contaminated with A. flavus and P. nordicum were analyzed.

\section{Results and Discussion}

\subsection{YMF and OMF Antifungal Activity}

Table 1 shows the antifungal activity in solid medium evidenced by the eight different extracts tested (YMF and OMF, without and with heat treatment, directly and after concentration) on the 14 mycotoxigenic fungi used in this study. 
Table 1. Antifungal activity evidenced by no autoclaved and autoclaved YMF and OMF against mycotoxigenic fungi employed in this study $(\mathrm{E}=$ Direct water extract; $\mathrm{L}=$ Lyophilized water extract). Calculation of antifungal activity: $8 \mathrm{~mm}$ diameter clearing zone $(+), 10 \mathrm{~mm}$ diameter clearing zone $(++)$, and more than $10 \mathrm{~mm}$ diameter clearing zone $(+++)$.

\begin{tabular}{ccccccccc}
\hline \multirow{2}{*}{ Strains } & \multicolumn{2}{c}{ YMF } & \multicolumn{2}{c}{ Autoclaved YMF } & \multicolumn{2}{c}{ OMF } & \multicolumn{2}{c}{ Autoclaved OMF } \\
\cline { 2 - 9 } & E & L & E & L & E & L & E & L \\
\hline P. camemberti (CECT 2267) & - & + & - & + & - & - & - & - \\
P. roqueforti (CECT 2905) & - & + & - & + & - & + & - & - \\
P. nordicum (CECT 2320) & - & + & - & - & - & - & - & - \\
P. commune (CECT 20767) & - & + & - & - & - & - & - & - \\
P. brevicopactum (CECT 2316) & - & + & - & - & - & - & - & - \\
P. expansum (CECT 2278) & - & + & - & - & - & - & - & - \\
P. chrysogenum (CECT 2668) & - & + & - & - & - & - & - & - \\
P. solitum (CECT 20818) & - & + & - & - & - & - & - & - \\
P. digitatum (CECT 2954) & - & + & - & - & - & - & - & - \\
A. parasiticus (CECT 2681) & + & + & - & - & - & - & - & - \\
A. flavus (ISPA 8111) & - & + & - & - & - & - & - & - \\
A. carbonarius (ISPA 5010) & - & + & - & - & - & + & - & - \\
F. verticiloides (ISPA 1044) & - & + & - & - & - & - & - & - \\
F. graminearum (CECT 20486) & - & - & - & - & - & - & - & - \\
\hline
\end{tabular}

The non-autoclaved YMF was active only against $A$. parasiticus, whereas the concentrated extract of the non-autoclaved YMF showed the highest antifungal activity on the strains tested, and in particular on 13 of the 14 fungi studied (Figure 2). The autoclaved YMF extract was negative against all fungal strains tested, while the concentrated YMF extract was active against $P$. camemberti and P. roqueforti.

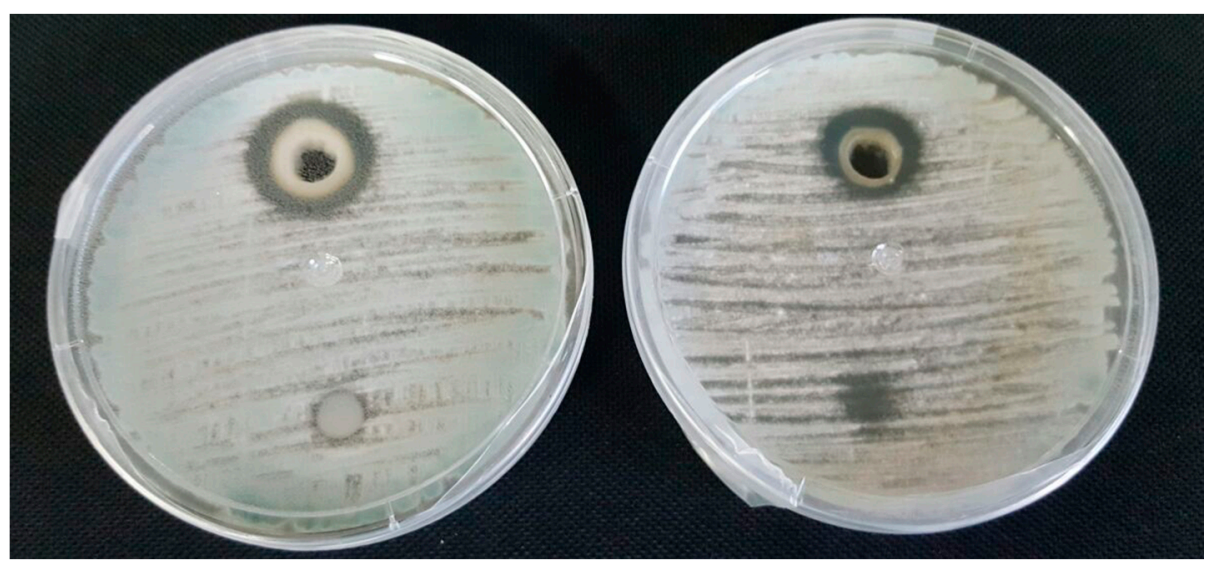

Figure 2. Antifungal activity of YMF lyophilized water extract against $P$. nordicum (CECT 2320) on PDA medium.

Considering the results of the antifungal activity of the OMF, only the concentrated extract of the non-autoclaved flour showed antifungal activity, against the strains of P. roqueforti and A. carbonarius. The other extracts of this bioactive ingredient tested did not shown any antifungal activity on the fungi tested at the incubation time employed. Considering the results of the antimicrobial activity on solid medium evidenced by the two matrices, the MIC and MFC of the concentrated extract of the no-autoclaved YMF was determined using the 96-well microplate assay.

As evidenced in Table 2, the MIC of the concentrated extract of YMF (non-autoclaved) ranged from 238.2 (P. camemberti) to $15,000 \mu \mathrm{g} / \mathrm{mL}$ (A. flavus, A. parasiticus and A. carbonarius). Important results of the antifungal activity of the YMF were also evidenced against $P$. roqueforti and $P$. digitatum with MIC data of 476.5 and $937.5 \mu \mathrm{g} / \mathrm{mL}$ respectively. Considering the MFC data, the YMF presented 
inhibition data that ranged from 1875 (P. nordicum, P. commune and P. brevicompactum) to $15,000 \mu \mathrm{g} / \mathrm{mL}$ (A. flavus, A. parasiticus and A. carbonarius). In general, the Aspergillus strains tested were more resistant to the YMF extract in comparison with the employed strains of Penicillium and Fusarium. Considering the Penicillium strains tested, the microorganisms that showed a very low resistance to the mustard extracts tested in this study were, $P$. camemberti and P. roqueforti, that showed the lower MIC values.

Table 2. MIC and MFC evidenced by the YMF lyophilized water extract in the mycotoxigenic fungi tested.

\begin{tabular}{ccc}
\hline Strain & MIC & MFC \\
& & $\mu \mathrm{g} / \mathrm{mL}$ \\
\hline P. camemberti (CECT 2267) & 238.2 & 1906.2 \\
P. roqueforti (CECT 2905) & 476.5 & 1906.2 \\
P. nordicum (CECT 2320) & 937.5 & 1875.5 \\
P. commune (CECT 20767) & 937.5 & 1875.5 \\
P. brevicopactum (CECT 2316) & 937.5 & 1875.5 \\
P. expansum (CECT 2278) & 1875 & 7500.2 \\
P. chrysogenum (CECT 2668) & 937.5 & 3750.5 \\
P. solitum (CECT 20818) & 3750 & 3750.5 \\
P. digitatum (CECT 2954) & 937.5 & 7500.3 \\
A. parasiticus (CECT 2681) & $>15,000$ & $>15,000$ \\
A. flavus (ISPA 8111) & $>15,000$ & $>15,000$ \\
A. carbonarius (ISPA 5010) & $>15,000$ & $>15,000$ \\
F. verticiloides (ISPA 1044) & 1875.5 & 3750.5 \\
\hline
\end{tabular}

Considering the other Penicillium strains, the MIC values detected for the YMF were always above $500 \mu \mathrm{g} / \mathrm{mL}$. The lyophilized extract of the YMF was also active against the strain of F. verticillioides, a fumonisins (FBs) producer, showing MIC and MFC values of 1875.5 and $3750.5 \mu \mathrm{g} / \mathrm{mL}$, respectively.

Several authors have tested the application of the ITCs as antimicrobial substances, both directly and using mustard flours. In the first case, Mañes et al. [12] studied the antifungal activity of allyl isothiocyanate (AITC) against two mycotoxigenic strains of the genera Aspergillus and Penicillium. Tests of antifungal activity in a solid medium showed that $5 \mu \mathrm{L}$ of AITC deposited inside a disc of sterile filter paper were sufficient to inhibit the growth of the A. parasiticus fungus seeded on the surface of a PDA medium plate. The amount of AITC needed to inhibit the growth of the fungus P. expansum was greater, specifically $25 \mu \mathrm{L} / \mathrm{L}$. Azaiez et al. [13] evaluated the antifungal activity of AITC, phenyl (PITC) and benzyl isothiocyanates (BITC) toward Fusarium mycotoxigenic strains. The ITCs employed in the study inhibited the growth of three mycotoxigenic Fusarium (Gibberella moniliformis), reducing $2.1-89.7 \%$ of the mycelium size depending on the time and the dose used (from 10 to $50 \mu \mathrm{L}$ ). The activity of ITCs against non-fungal pathogenic microorganisms transmitted by food has also been studied. Ekanayake et al. [11] demonstrated that $\rho$-HBIT had significant bactericidal activity against E. coli, S. aureus, C. jejuni, P. aeruginosa, S. enteritidis, L. monocytogenes, S. boydii and C. perfringens at a dose of $0.35-2.13 \mathrm{mM}$.

Other studies have demonstrated the antimicrobial capacity of mustard flours directly instead of ITCs. Kanemaru and Miyamoto [14] compared the antimicrobial effects of mustard and purified AITC at equal concentrations of AITC. They found that mustard was more effective against E. coli than purified AITC. They found that $0.1 \%$ mustard with $9.4 \mu \mathrm{g} / \mathrm{mL}$ of AITC was able to inhibit the growth of $E$. coli in culture medium within $24 \mathrm{~h}$ but $12.3 \mu \mathrm{g} / \mathrm{mL}$ of purified AITC was required to achieve the same level of inhibition. Mayerhauser [15] found that retail style mustards eliminated $6 \log 10$ of E. coli $\mathrm{O} 157: \mathrm{H} 7$ from trypticase soy broth within a few hours at refrigerator or room temperature. More recently, Rhee et al. [16] showed that mustard flour alone or with acetic acid reduced $6 \log 10$ of E. coli O157:H7 to $0.3 \log 10$ in $24 \mathrm{~h}$ at room temperature.

\subsection{Inhibition of Fungal Growth}

The growing demand for safe foods without synthetic chemical preservatives has prompted scientists to investigate the effects of natural compounds against the growth of several pathogenic 
microorganisms $[8,17,18]$. The evaluation of the use of YMF in breads inoculated with $A$ flavus and $P$. nordicum compared with breads produced with sodium propionate, the classical commercial additive for bakery products, showed that none of the treatments used completely reduced the A. flavus and P. nordicum growth (Figure $3 \mathrm{a}, \mathrm{b}$ ). However, some preservative treatments applied in this study were able to reduce the fungal growth of the mycotoxigenic fungi tested in comparison to the control experiment.

a)

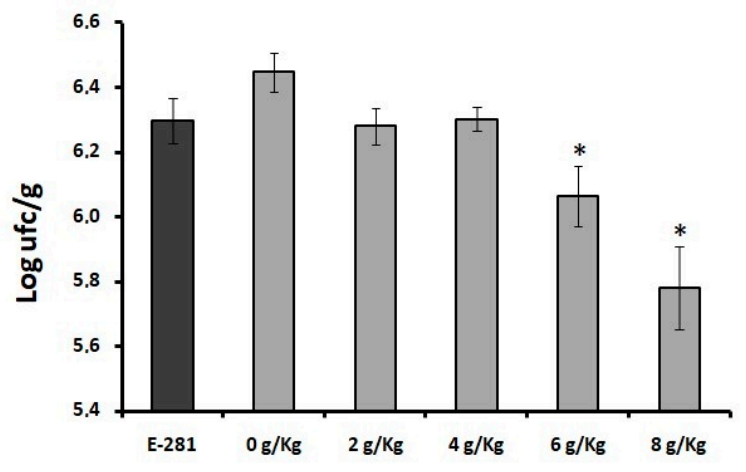

b)

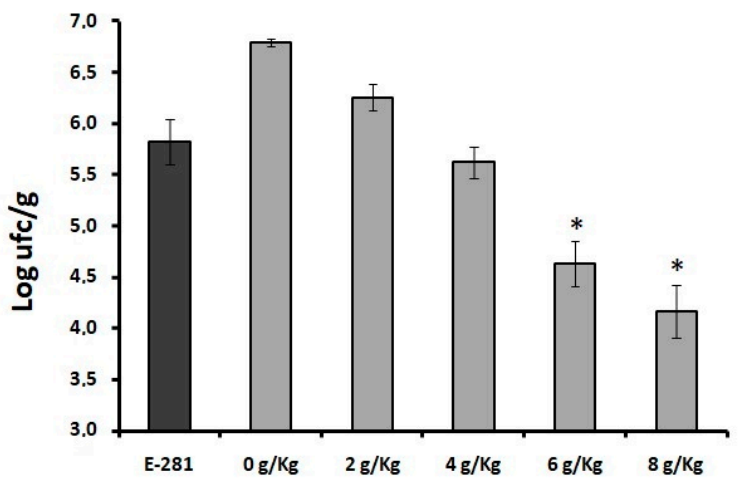

Figure 3. Population of (a) A. flavus and (b) P. nordicum in bread loaves treated with natural (YMF) and synthetic commercial (E-281) preservatives. Significantly different from the commercial control, $\left.p \leq 0.05\left(^{*}\right), p \leq 0.001\left(^{* *}\right), p \leq 0.0001{ }^{* * *}\right)$.

As shown in Figure 3a, the bread contaminated with A. flavus, stored during 10 days and produced with sodium propionate presented a fungal contamination of $630 \mathrm{Log} / \mathrm{UFC} / \mathrm{g}$ and no statistically differences in the microorganism growth were observed in the bread treated with 2 and $4 \mathrm{~g} / \mathrm{kg}$ of $\mathrm{YMF}$ (628 and $630 \mathrm{Log} / \mathrm{UFC} / \mathrm{g}$ respectively). In the bread treated with $6 \mathrm{~g} / \mathrm{kg}(606 \mathrm{Log} / \mathrm{UFC} / \mathrm{g})$ and $8 \mathrm{~g} / \mathrm{kg}$ (578 Log/UFC/g) a significant difference on the A. flavus was observed in comparison with the bread treated with the E-281, with a reduction of 0.24 and $0.52 \mathrm{Log} / \mathrm{UFC} / \mathrm{g}$ respectively $(47$ and $67 \% \mathrm{UFC} / \mathrm{g}$, respectively).

Related to the results of the $P$. nordicum growth in the loaf bread produced with sodium propionate and with the YMF, the results of the fungal growth along the incubation period are shown in Figure $3 \mathrm{~b}$. In particular the bread loaves produced with sodium propionate (at 10 days storage) presented a fungal contamination of $582 \mathrm{Log} \mathrm{UFC} / \mathrm{g}$, whereas in the bread loaves treated with 2 and $4 \mathrm{YMF}$, the level of contamination was 626 and $562 \mathrm{Log} \mathrm{UFC} / \mathrm{g}$ respectively.

The bread treated with 6 and $8 \mathrm{~g} / \mathrm{kg}$ of the YMF presented a statistically difference of P. nordicum growth of 463 and $416 \mathrm{Log}$ UFC/g respectively in comparison with the bread produced with the E-281, with a reduction of the fungal growth of the 1.19 and $1.66 \mathrm{Log} / \mathrm{UFC} / \mathrm{g}$ (94 and 97\% UFC/g respectively).

The use of ITCs generated from mustard flours has been studied by several authors. Quiles et al. [19] tested active packaging devices containing AITC or OMF + water to inhibit the growth of A. parasiticus in fresh pizza doughs after 30 days of inoculation. The antimicrobial activities were compared with 
a control group (non-antimicrobial treatment) and a group added with a commercial preservative (sodium propionate). The growth of $A$. parasiticus was inhibited after 30 days with AITC on filter paper at $5 \mu \mathrm{L} / \mathrm{L}$ and $10 \mu \mathrm{L} / \mathrm{L}$, and on of OMF at $850 \mathrm{mg}+1 \mathrm{~mL}$ of water. The use of yellow mustard as an ingredient has been reported for acid food matrices against pathogenic microorganisms. Graumann and Holley [20] demonstrated that the $p$-HBITC generated in situ by including ground yellow mustard powder as an ingredient in dry-fermented sausages inhibited the growth of E. coli O157:H7.

\subsection{Mycotoxin Reduction}

Mean recovery of fortified bread loaves samples $(n=3)$ at three different levels of $\mathrm{AFB}_{1}$ and $\mathrm{AFB}_{2}(5,10$ and $15 \mu \mathrm{g} / \mathrm{kg})$ was of $84.6 \pm 3.6 \%$ and $88.2 \pm 3.3 \%$, respectively. The values obtained for recovery and relative standard deviations of the method used agree with the EU Commission Directive 2006/401/EC for methods to analyze bioactive compounds in foodstuffs [21]. Intra-day $(n=5)$ and inter-day ( 5 different days) variation values were 2.5 and $8.6 \%$, respectively. These values are below $15 \%$, which is the maximum variation for certification exercises of bioactive compounds. The detection limit (LOD) and the limit of quantification (LOQ) values were 0.05 and $0.15 \mu \mathrm{g} / \mathrm{kg}$, respectively. Linearity, plotted as DAD response area against concentration estimated for the matrix matched standards, and matrix effects were studied using standard solutions and matrix matched calibrations.

Calibration curves were built at eight different mycotoxins levels, from LOQ to 100 times LOQ (from 0.1 to $300 \mathrm{ppm}$ ). Each level was prepared in triplicate. Slopes of standard solutions were compared with those obtained in matrix matched standards to assess the possible matrix effect on the chromatographic response. The results obtained showed that the matrix effect calculated for $\mathrm{AFB}_{1}$ and $\mathrm{AFB}_{2}$ were of 87.4 and $89.6 \%$ respectively.

The analysis of AFs in the bread inoculated with A. flavus (Figure 4), evidenced only the production by the mycotoxigenic fungi of the $\mathrm{AFB}_{1}$. Analyzing the results shown in the figure, the breads produced with 2,4 and $6 \mathrm{~g} / \mathrm{kg}$ of YMF and contaminated with $A$. flavus, presented amounts of the $\mathrm{AFB}_{1}$ not different from the statistical point of view in comparison with the control experiment, whereas in bread loaves treated with $8 \mathrm{~g} / \mathrm{kg}$ of $\mathrm{YMF}$ no $\mathrm{AFB}_{1}$ concentration was detected, confirming the antifungal potential of the ingredient employed.

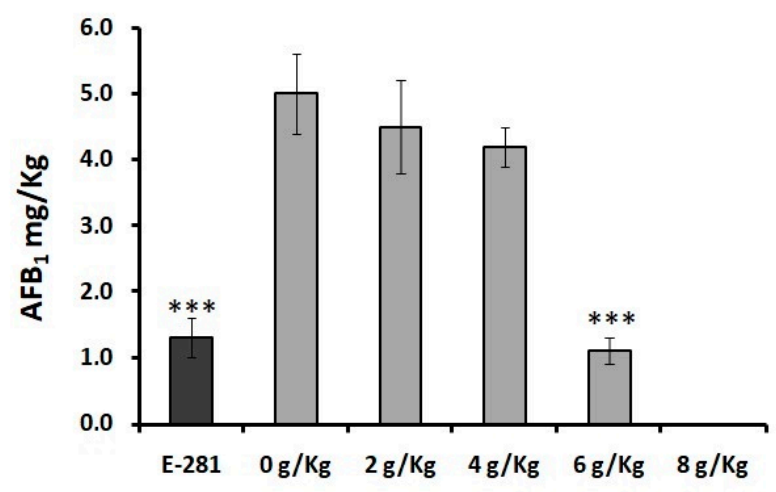

Figure 4. Concentration and reduction rate of $\mathrm{AFB}_{1}$ present in bread loaves contaminated with A. flavus and treated with several preserving treatments (Black = synthetic commercial E-281 preservative, Grey $=$ Different doses of natural YMF) at 10 days of incubation. Significantly different from the bread without treatment, $\left.p \leq 0.05\left(^{*}\right), p \leq 0.001{ }^{* *}\right), p \leq 0.0001\left(^{* * *}\right)$.

The amount of the $\mathrm{AFB}_{1}$ detected in the control experiment (a loaf of bread produced without any preservative ingredient) was of $5 \mathrm{mg} / \mathrm{kg}$, whereas the loaves treated with sodium propionate showed a level of contamination of $\mathrm{AFB}_{1}$ of $1.3 \mathrm{mg} / \mathrm{kg}$. The bread loaves treated with $6 \mathrm{~g} / \mathrm{kg}$ of $\mathrm{YMF}$ presented $1.1 \mathrm{mg} / \mathrm{kg}$ of the $\mathrm{AFB}_{1}$ with a percentage of reductions of $\mathrm{AFB}_{1}$ in comparison with the 
control of the $78 \%$. Mycotoxin production by P. nordicum was not observed in bread loaf contaminated with this fungus.

Saladino et al. [22] used ITCs derived from OMF and YMF (0.1, 0.5 and $1 \mathrm{~g}$ of flour) to avoid the production of AFs in piadina (a typical Italian flatbread) contaminated with $A$. parasiticus. In addition, the antifungal activity of the isothiocyanates toward $A$. parasiticus was also evaluated. The mustard flours employed in this study inhibited the growth of A. parasiticus, reducing the mycelium size by $12.2-80.6 \%$. The ITC produced in situ also reduced the AFs biosynthesis in Italian piadina. The use of YMF reduced the AFs content by 41-69.2\%. The same authors [23] investigated the use the ITCs generated by the addition of water to OMF and YMF for the reduction of the formation of mycotoxins produced by strains of the genus Penicillium as P. expansum. The patulin reduction (PAT) evidenced in the treated samples varied from 80 to $100 \%$.

Hontanaya et al. [24] studied the reduction of AFs produced by A. parasiticus in nuts using ITCs (AITC and p-hydroxy benzyl isothiocyanate (p-HBITC)) produced by the enzymatic hydrolysis of glucosinolates sinigrin (SG) and sinalbin (SA) present in OMF and YMF. The reduction of $\mathrm{AFB}_{1}, \mathrm{~B}_{2}$, $\mathrm{G}_{1}$ and $\mathrm{G}_{2}$ observed ranged from 83.1 to $87.2 \%$ and from 27.0 to $32.5 \%$ after nuts exposure to AITC and p-HBITC respectively.

\subsection{Shelf Life Analysis}

Considering the results of the shelf life improvement of the bread loaves treated with the sodium propionate and with different concentrations of YMF, it's possible to underline several important data.

In particular, considering the results on the mycotoxigenic fungi $A$. flavus, the control bread (bread produced with any preservative compound or ingredient) showed a visible fungal growth at 2 days of incubation (Table 3a), whereas the bread treated with sodium propionate (E-281), presented an evidence of the fungal growth at 3 days of incubation. The bread loaves produced with 2 and $4 \mathrm{~g} / \mathrm{kg}$ of the YMF presented the same pattern of the A. flavus growth recorded for the experiment carried out with the E-281. The bread loaves produced with $8 \mathrm{~g} / \mathrm{kg}$ of YMF, did not shown any fungal growth during the incubation period used in this study.

Table 3. Shelf life, monitored in days, of the bread loaves treated with 4 different concentrations of the YMF and contaminated with (a) Aspergillus flavus (ISPA 8111) and (b) Penicillium nordicum (CECT 2320), in comparison with the commercial control produced with the additive E-281, and with a loaf bread prepared without any antimicrobial treatment.

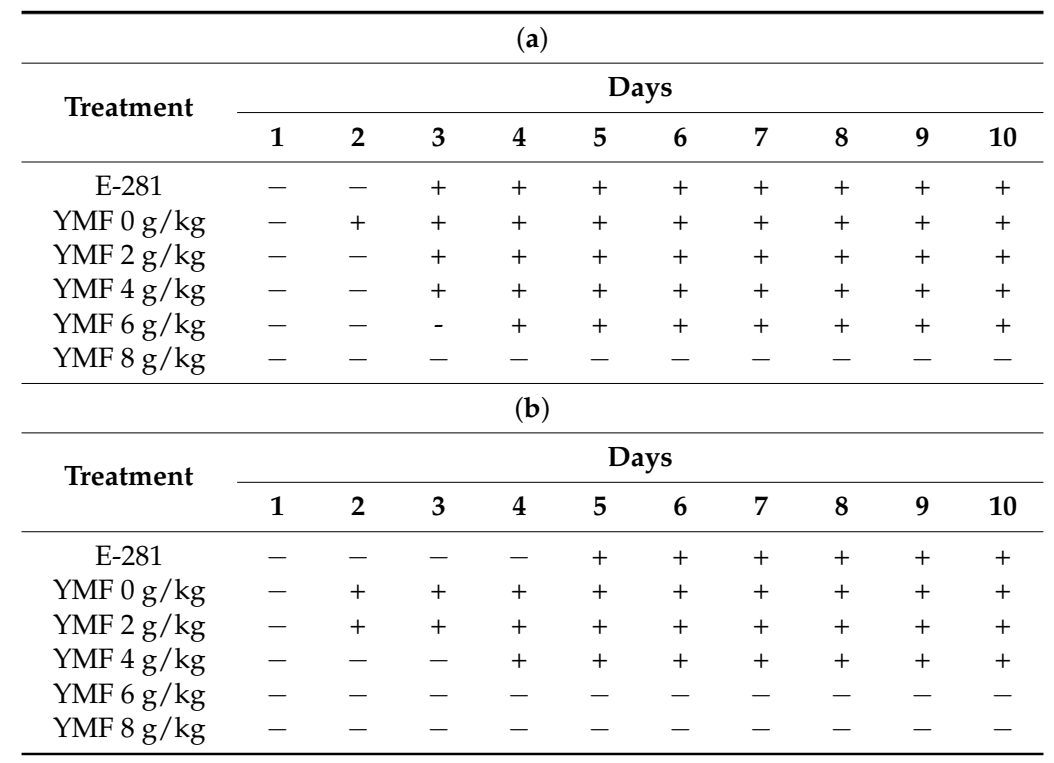


Considering the shelf life improvement observed in the bread loaves contaminated with P. nordicum and treated with the antimicrobial ingredients tested (Table $3 b$ ), the bread produced with E-281, presented a visible fungal growth at 5 days of incubation, while the control bread and the loaves produced with 2 and $4 \mathrm{~g} / \mathrm{kg}$ of YMF, presented a microbial growth at 2 and 4 days respectively. In the breads baked using 6 and $8 \mathrm{~g} / \mathrm{kg}$ of $\mathrm{YMF}$, it was not observed any $P$. nordicum growth during the incubation period used (Figure 5).

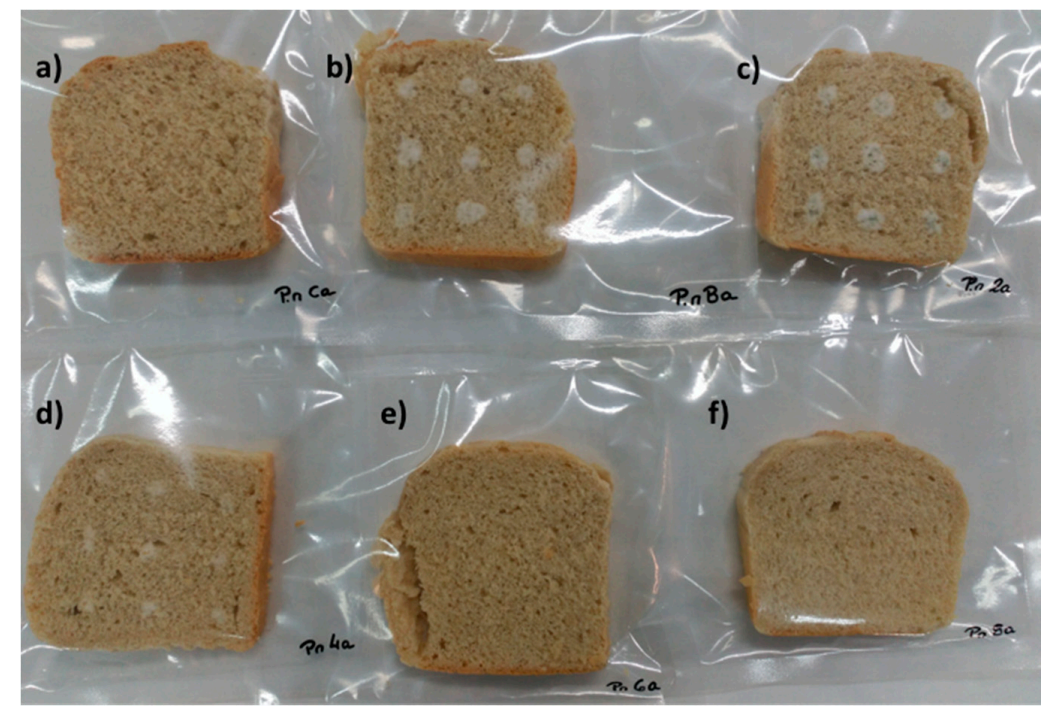

Figure 5. Bread loaves contaminated with P. nordicum and treated with (a) $2.0 \mathrm{~g} / \mathrm{kg}$ of sodium propionate (E-281); (b) not treated; (c) $2 \mathrm{~g} / \mathrm{kg}$ of YMF; (d) $4 \mathrm{~g} / \mathrm{kg}$ of YMF; (e) $6 \mathrm{~g} / \mathrm{kg}$ of YMF and (f) $8 \mathrm{~g} / \mathrm{kg}$ of YMF, after 10 days of incubation.

\section{Materials and Methods}

\subsection{Chemicals and Microorganisms}

AFs $B_{1}, B_{2}, G_{1}, G_{2}$, sinigrin (98\% purity), formic acid (HCOOH), sodium propionate, tetrabutylammonium hydrogen sulfate (TBA), ammonium formate, and sodium chloride $(\mathrm{NaCl})$ were obtained from Sigma Aldrich (St. Louis, MO, USA). YMF and OMF were provided by G.S. Dunn Dry Mustard Millers (Hamilton, ON, Canada). Methanol was purchased from Fisher Scientific (Rockingham County, NH, USA). Deionized water ( $<\mathrm{M} 18 \mathrm{MX} \mathrm{cm}$ resistivity) was obtained from a Milli-Q water purification system (Millipore, Bedford, MA, USA). Chromatographic solvents and water were degassed for 20 min using a Branson 5200 (Branson Ultrasonic Corp., Danbury, CT, USA) ultrasonic bath. The strains of Aspergillus parasiticus CECT 2681, Penicillium camemberti CECT 2267, Penicillium roqueforti CECT 2905, Penicillium nordicum CECT 2320, Penicillium commune CECT 20767, Penicillium brevicopactum CECT 2316, Pencillium expansum CECT 2278, Penicillium chrysogenum CECT 2668, Penicillium solitum CECT 20818, Penicillium digitatum CECT 2954 and Fusarium graminearum CECT 20486 were obtained from the the Spanish Type Culture Collection (CECT, Valencia, Spain). The strains of Aspergillus flavus ISPA 8111, Aspergillus carbonarius ISPA 5010 and Fusarium verticiloides ISPA 12044 were obtained from the Institute of Sciences of Food Production (ISPA-CNR, Bari, Italy). Buffered peptone water, potato dextrose agar (PDA) and potato dextrose broth (PDB) were acquired from Oxoid (Madrid, Spain).

\subsection{Extraction of the Water-Soluble Components from Mustard Flours}

The water-soluble components of YMF and OMF were extracted using the method of Hontanaya et al. [24] with some modifications. Flour (5 g) was placed in a $50 \mathrm{~mL}$ glass tube and 
autoclaved at $115^{\circ} \mathrm{C}$ for $15 \mathrm{~min}$ to inactivate the enzyme myrosinase. This matrix was used to confirm that the possible antimicrobial activity of the mustard flours was due to the ITCs. Experiments with untreated mustard flours were carried out directly using the flour matrices. $25 \mathrm{~mL}$ of distilled water was introduced into the same tube and the extraction was carried out using an UltraTurrax T18 basic mixer (Ika, Staufen, Germany) for $3 \mathrm{~min}$ at 11,000 rpm. The mixture was centrifuged at $2500 \mathrm{rpm}$ for $5 \mathrm{~min}$ at $4{ }^{\circ} \mathrm{C}$ and filtered through a filter paper (Whatman No. 4) in $50 \mathrm{~mL}$ tubes with screw cap. Finally, the extract was filtered again through a $0.22 \mu \mathrm{M}$ filter and kept under refrigeration at $4{ }^{\circ} \mathrm{C}$. To test also the antimicrobial activity of the concentrated mustard extracts, half of them were lyophilized by depositing $25 \mathrm{~mL}$ of extract in $100 \mathrm{~mL}$ plastic containers in a Virtis SP SCIENTIFIC Sentinel 2.0 lyophilizer (Warminster, PA, USA).

\subsection{Antifungal Activity Tests on Solid Medium}

The method used to evaluate the antimicrobial activity of the components of water-soluble mustard meal was as follows. The lyophilized extracts of YMF and OMF were previously suspended in $1 \mathrm{~mL}$ of sterile water obtaining a concentrated extract. The fungi to be analyzed were grown on the surface with a cotton swab in $9 \mathrm{~mm}$ Petri dishes prepared with $20 \mathrm{~mL}$ of PDA. In each plate, $10 \mu \mathrm{L}$ in surface and $100 \mu \mathrm{L}$ in pre-prepared agar wells were added. The triplicates of each plate were incubated at $28^{\circ} \mathrm{C}$ observing the development of fungal growth at $48 \mathrm{~h}$.

\subsection{Antifungal Activity Tests on Liquid Medium}

The assay was performed in 96-well sterile microplates, using the modified method of Siah et al. [25]. The tests of antifungal activity in liquid medium were not carried out with OMF due to the results obtained in the tests in solid medium. The first column served as control of the medium. For this, $200 \mu \mathrm{L}$ of PDB were added in each of the wells to verify the absence of contamination of the medium. The next column consisted of a control of the microorganism, to verify its viability, so $100 \mu \mathrm{L}$ of PDB was deposited. The remaining (3-12) contained $100 \mu \mathrm{L}$ of lyophilized YMF extract resuspended at doses between 30 to $15,000 \mathrm{ppm}$. Each well was inoculated with $100 \mu \mathrm{L}$ of a $5 \times 10^{4}$ spores/mL suspension in PDB of the mycotoxigenic fungi described in the paragraph 2.1. The negative control consisted of inoculated medium without any treatment. The plates were incubated at $25^{\circ} \mathrm{C}$ for $72 \mathrm{~h}$ in the dark. Four wells were used for each assay of each fungus ( 2 fungi per 96-well plate) and the experiments were performed in triplicate.

The minimum inhibitory concentration (MIC) was defined as the lowest concentration of the YMF extract, where the fungi did not show any visible growth. For the determination of the minimum fungicidal concentration (MFC), after determining the MIC, the concentrations corresponding to the inhibitory and to higher concentrations, as well as the controls, were subcultured on PDA plates. After $72 \mathrm{~h}$ of incubation at $25^{\circ} \mathrm{C}$, MFCs' readings were made being MFC the lowest extract concentration in which a visible growth of the subculture was prevented.

\subsection{Baking and Bread Treatment}

The bread recipe included $600 \mathrm{~g}$ of wheat flour, $20 \mathrm{~g}$ of sucrose, $10 \mathrm{~g}$ of NaCl, $40 \mathrm{~g}$ of yeast for bakery products (Levital, Spain) and $250 \mathrm{~mL}$ of tap water. The commercial control was prepared adding to the ingredients sodium propionate (E-281) at $2 \mathrm{~g} / \mathrm{kg}$ that is a common preservative used for loaf bread production in Spain. The studied breads were produced adding to the basic ingredients YMF at the concentration of $0,2,4,6$ and $8 \mathrm{~g} / \mathrm{kg}$ of dry ingredient in the dough. The ingredients were kneaded manually for 10 min and the dough produced was left rising for $1 \mathrm{~h}$ at room temperature. Baking was performed at $230^{\circ} \mathrm{C}$ for $30 \mathrm{~min}$ in a deck oven (MIWE, Arnstein, Germany). The oven was pre-steamed ( $300 \mathrm{~mL}$ of water) before cooking. The loaves were kept for $30 \mathrm{~min}$ on cooling racks at room temperature. Loaves were cut in slices of $30 \mathrm{~g}$ each. The slices were inoculated with $100 \mu \mathrm{L}$ of a suspension containing $1 \times 10^{5}$ conidia/mL of $A$. flavus or $P$. nordicum. Conidial concentration was measured by optical density at $600 \mathrm{~nm}$ and adjusted to $10^{5}$ conidia/mL in PDB as reported 
by Kelly et al. [26] and introduced in 1 L plastic trays. All plastic trays were closed hermetically and incubated at room temperature for 10 days. Each day until the analysis the bread slices were examined to determine the visible fungal growth and the shelf life evaluation. Then, all packages were opened, and samples contaminated with A. flavus were used to determine the $\mathrm{AFB}_{1}$ content by liquid chromatography coupled to mass spectrometry in tandem (LC-MS/MS). Each bread was made in triplicate and from each bread 3 slices were analyzed $(n=9)$.

\subsection{Aflatoxins Extraction}

AFs extraction was performed using the method described by Hontanaya et al. [24] Briefly, the bread slices were finely grounded with a blender (Oster Classic grinder, Oster, Valencia, Spain) and $5 \mathrm{~g}$ samples were placed in a $50 \mathrm{~mL}$ plastic tube. Then, $0.5 \mathrm{~g}$ of sodium chloride $(\mathrm{NaCl})$ and $25 \mathrm{~mL}$ of a methanol/water $(80: 20, v / v)$ mixture were added. Samples were homogenized using an Ultra Ika T18 basic UltraTurrax (Staufen, Germany) for $3 \mathrm{~min}$. The mixture was centrifuged at $4500 \mathrm{rpm}$ for $5 \mathrm{~min}$ and the supernatant was evaporated to dryness with a Rotavapor R-200 (Büchi, Postfach, Switzerland). The residue was re-dissolved in $1 \mathrm{~mL}$ of extraction solvent, filtered through a $0.22 \mu \mathrm{m}$ syringe filter and injected to the LC-MS/MS system.

Commission Decision 2002/657/EC [27] and 401/2006/EC [21] were used as guidelines for the validation studies. All the parameters were evaluated by spiking blank samples $(5,10$ and $15 \mu \mathrm{g} / \mathrm{kg}$ of $\mathrm{AFB}_{1}$ and $\mathrm{AFB}_{2}$ ), which were left to equilibrate overnight before the analysis. For identification purposes, retention time of compound in standards and samples were compared at tolerance of $70.5 \%$. Method performance characteristics such as linearity, limits of detection (LOD), limits of quantitation (LOQ), matrix effect, recovery, repeatability and reproducibility were evaluated for sinigrin.

\subsection{AFs Identification and Quantification by LC-MS/MS}

LC-MS/MS analyses were performed with a system consisting of Agilent 1200 chromatograph (Agilent Technologies, Palo Alto, CA, USA) coupled to a 3200 QTRAP mass spectrometer (Applied Bio-systems, AB Sciex, Foster City, CA, USA) equipped with a turbo ionspray electrospray ionisation (ESI) interface. The instrument data were collected and processed using the Analyst version 1.5.2 software (AB Sciex, Foster City, CA, USA). Separation of analytes was performed using a reversed-phase analytical column (Gemini C18 column, $150 \times 2 \mathrm{~mm}$, I.D. $3 \mu \mathrm{m}$ particle size), equipped with a security guard cartridge C18 $(4 \times 2 \mathrm{~mm}$, I.D.; $5 \mu \mathrm{m})$ all from Phenomenex (Madrid, Spain). The mobile phases used were: Water with $0.1 \%$ of formic acid and $5 \mathrm{mM}$ ammonium formate (Phase A) and methanol with $0.1 \%$ of formic acid and $5 \mathrm{mM}$ ammonium formate (Phase B). The elution gradient was established initially with $10 \%$ eluent B. It was increased to $80 \%$ in $1.5 \mathrm{~min}$ and was kept constant until 4 th min. Then it increased to $90 \%$ for $6 \mathrm{~min}$. Subsequently, it was increased to $100 \%$ until the 14th minute and then reduced to $50 \%$ at the minute 17. Afterwards the initial conditions were maintained for $5 \mathrm{~min}$. The flow rate was $0.25 \mathrm{~mL} \mathrm{~min}$ and MS/MS were achieved in the selected reaction monitoring (SRM) mode using ESI in positive mode. For LC-MS/MS analysis, scheduled SRM was used with a $120 \mathrm{~s}$ SRM detection window and $1 \mathrm{~s}$ of target scan time. The applied parameters were: ion spray voltage, $5500 \mathrm{~V}$; source temperature, $450{ }^{\circ} \mathrm{C}$; curtain gas, 20; ion source gas 1 (sheath gas), 50 psi; ion source gas 2 (drying gas), 55 psi. Nitrogen served as nebulizer and collision gas. The ionization and fragmentation parameters used for the detection and quantification of the AFs were set according to Liu et al [16].

\subsection{Determination of the Fungal Population}

After incubation, each slice was weighed and transferred to a sterile plastic bag with sterile peptone water (Oxoid) in a 1:10 dilution and homogenized with a stomacher (IUL, Barcelona, Spain) during $30 \mathrm{~s}$. The mixture was serially diluted in sterile plastic tubes containing $0.1 \%$ peptone water. Aliquots of $100 \mu \mathrm{l}$ were seeded in acidified PDA (pH 3.5) (Insulab, Valencia, Spain) and the plates were incubated at $25^{\circ} \mathrm{C}$ for 7 days before the microbial count [17]. 


\section{Conclusions}

The present study demonstrated the capacity of YMF extracts to inhibit or reduce the growth of several fungal strains belonging to Aspergillus, Penicillium and Fusarium species. Moreover, YMF used as an ingredient in bread preparation can significantly retard and reduce A. flavus and P. nordicum growth, improve the shelf life of the bread and reduce $\mathrm{AFB}_{1}$ naturally produced on substrate by A. flavus, so this study shows that YMF can be potentially used as natural preservative for bakery products which are commonly contaminated by Aspergillus and Penicillium species. YMF could be a potential substitute to conventional preservatives used in bread satisfying the growing demand of the consumer for natural products free from common chemical preservatives.

Author Contributions: The authors J.M.Q., R.T. and F.L. carried out the experiments related on the antimicrobial activity of the mustard flours, whereas J.M. and G.M. carried out the application part in bread and also the analystical part on the determination and quantification of the AFs. J.M. and G.M. designed the experiments.

Acknowledgments: The Ministry of Economy and Competitiveness (AGL2016-77610R) and the pre PhD program of University of Valencia (Atracció de Talent) supported the research activity carried out in this manuscript.

Conflicts of Interest: The authors declare that have no conflicts of interest.

\section{References}

1. EFSA-European Food Safety Authority. Opinion of the scientific panel on contaminants in the food chain on a request from the commission related to the potential increase of consumer health risk by a possible increase of the existing maximum levels for aflatoxins in almonds, hazelnuts and pistachios and derived products. EFSA J. 2007, 446, 1-127.

2. Peterson, S.W.; Ito, Y.; Horn, B.W.; Goto, T. Aspergillus bombycis, a new aflatoxigenic species and genetic variation in its sibling species, Aspergillus nomius. Mycologia 2001, 93, 689-703. [CrossRef]

3. Coulombe, R.A. Aflatoxins. In Mycotoxins and Phytoalexins; Sharma, R.P., Salunkhe, D.K., Eds.; CRC Press: Boca Raton, FL, USA, 1991; pp. 103-143.

4. Amano, K. Monographs on the evaluation of carcinogenic risks to humans. In A Review of Biological Agents for Human Carcinogens; International Agency for Research on Cancer: Lyon, France, 2012.

5. Pal Vig, A.; Rampal, G.; Thind, T.S.; Arora, S. Bio-protective effects of glucosinolates. LWT Food Sci. Technol. 2009, 42, 1561-1572.

6. Tunc, S.; Chollet, E.; Chalier, P.; Preziosi-Belloy, L.; Gontard, N. Combined effect of volatile antimicrobial agents on the growth of Penicillium notatum. Int. J. Food Microbiol. 2007, 113, 263-270. [CrossRef] [PubMed]

7. Sellam, A.; Iacomi-Vasilescu, B.; Hudhomme, P.; Simoneau, P. In vitro antifungal activity of brassinin, camalexin and two isothiocyanates against the crucifer pathogens Alternaria brassicicola and Alternaria brassicae. Plant Pathol. 2007, 56, 296-301. [CrossRef]

8. Nadarajah, D.; Han, J.H.; Holley, R.A. Inactivation of Escherichia coli O157:H7 in packaged ground beef by allyl isothiocyanate. Int. J. Food Microbiol. 2005, 99, 269-279. [CrossRef] [PubMed]

9. Lara-Lledó, M.; Olaimat, A.; Holley, R.A. Inhibition of Listeria monocytogenes on bologna sausages by an antimicrobial film containing mustard extract or sinigrin. Int. J. Food Microbiol. 2012, 156, 25-31. [CrossRef] [PubMed]

10. Chen, W.; Jin, T.Z.; Gurtler, J.B.; Geveke, D.J.; Fan, X. Inactivation of Salmonella on whole cantaloupe by application of an antimicrobial coating containing chitosan and allyl isothiocyanate. Int. J. Food Microbiol. 2012, 155, 165-170. [CrossRef] [PubMed]

11. Ekanayake, A.; Kester, J.J.; Li, J.J.; Zehentbauer, G.N.; Bunke, P.R.; Zent, J.B. Isogard ${ }^{\mathrm{TM}}$ : A natural anti-microbial agent derived from white mustard seed. ActaHorticulturae 2006, 709, 101-108. [CrossRef]

12. Mañes, L.; Luciano, F.B.; Mañes, J.; Meca, G. In vitro antifungal activity of allyl isothiocyanate (AITC) against Aspergillus parasiticus and Penicillium expansum and evaluation of the AITC estimated daily intake. Food Chem. Toxicol. 2015, 83, 293-299. [CrossRef] [PubMed]

13. Azaiez, I.; Meca, G.; Manyes, L.; Luciano, F.B.; Fernández-Franzón, M. Study of the cemical reduction of the fumonisins toxicity using allyl, benzyl and phenyl isothiocyanate in model solution and in food products. Toxicon 2013, 63, 137-146. [CrossRef] [PubMed] 
14. Kanemaru, K.; Miyamoto, T. Inhibitory effects on the growth of several bacteria by brown mustard and allylisothiocyanate. Nippon Shokuhin Kogyo Gakkaishi 1990, 37, 823-829. (In Japanese) [CrossRef]

15. Mayerhauser, C.M. Survival of enterohemorrhagic Escherichia coli O157:H7 in retail mustard. J. Food Prot. 2001, 64, 783-787. [CrossRef] [PubMed]

16. Rhee, M.S.; Dougherty, R.H.; Kang, D.H. Combined effects of mustard flour, acetic acid, and salt against Escherichia coli O157:H7 stored at 5 and $22^{\circ} \mathrm{C}$. J. Food Prot. 2002, 65, 1632-1635. [CrossRef] [PubMed]

17. Luciano, F.B.; Belland, J.; Holley, R.A. Microbial and chemical origins of the bactericidal activity of thermally treated yellow mustard powder to ward Escherichia coli O157:H7 during dry sausage ripening. Int. J. Food Microbiol. 2011, 145, 69-74. [CrossRef] [PubMed]

18. Cordeiro, R.P.; Luciano, F.B.; Holley, R.A. Evaluation of deodorized yellow mustard concentrations for control of Escherichia coli O157:H7 viability in dry fermented sausage. Food Control 2013, 33, 20-24. [CrossRef]

19. Quiles, J.M.; Manyes, L.; Luciano, F.; Manes, J.; Meca, G. Antimicrobial compound allylisothiocyanate against the Aspergillus parasiticus growth and its aflatoxins production in pizza crust. Food Chem. Toxicol. 2015, 83, 222-228. [CrossRef] [PubMed]

20. Graumann, G.; Holley, R.A. Inhibition of E. coli O157:H7 in ripening dry fermented sausage by ground yellow mustard. J. Food Prot. 2008, 71, 486-493. [CrossRef] [PubMed]

21. European Union. European Commission, Regulation (EC) No 401/2006 of 23 February 2006 laying down the methods of sampling and analysis for the official control of the levels of mycotoxins in foodstuffs. Off. J. Eur. Union 2006, L70, $12 \mathrm{e} 34$.

22. Saladino, F.; Bordin, K.; Manyes, L.; Luciano, F.B.; Manes, J.; Fernandez-Franzon, M.; Meca, G. Reduction of the aflatoxins $\mathrm{B}_{1}, \mathrm{~B}_{2}, \mathrm{G}_{1}$ and $\mathrm{G}_{2}$ in Italian piadina by isothiocyanates. LWT Food Sci. Technol. 2016, 70, 302-308. [CrossRef]

23. Saladino, F.; Manyes, L.; Luciano, F.B.; Manes, J.; Fernandez-Franzon, M.; Meca, G. Bioactive compounds from mustard flours for the control of patulin production in wheat tortillas. LWT Food Sci. Technol. 2016, 66, 101-107. [CrossRef]

24. Hontanaya, C.; Meca, G.; Luciano, F.B.; Manes, J.; Font, G. Inhibition of the Aflatoxins $B_{1}, B_{2}, G_{1}$, and $G_{2}$, production by Aspergillus parasiticus in nuts using yellow and oriental mustard flours. Food Control 2015, 47, 154-160. [CrossRef]

25. Siah, A.; Deweer, C.; Morand, E.; Reignault, P.; Halama, P. Azoxystrobin resistance of French Mycosphaerella graminicola strains assessed by four in vitro bioassays and byscreening of G143A substitution. Crop Prot. 2010, 29, 737-743. [CrossRef]

26. Kelly, S.; Grimm, L.H.; Bendig, C.; Hempel, D.C.; Krull, R. Effects of fluid dynamic induced shear stress on fungal growth and morphology. Process. Biochem. 2006, 41, 2113-2117. [CrossRef]

27. European Union. European Commission, Decision of 12 August 2002 implementing Council Directive 96/23/EC concerning the performance of analytical methods and the interpretation of results. Off. J. Eur. Union 2002, L221, 8e34.

Sample Availability: Samples of the compounds are not available from the authors.

(C) 2018 by the authors. Licensee MDPI, Basel, Switzerland. This article is an open access article distributed under the terms and conditions of the Creative Commons Attribution (CC BY) license (http:/ / creativecommons.org/licenses/by/4.0/). 